\title{
ON PRINCIPAL TORUS BUNDLES OVER A HOMOGENEOUS CONTACT MANIFOLD
}

\author{
Yosuke OGAWA
}

(Received May 14, 1964)

Introduction. W. M. Boothby and H. C. Wang [3] have studied a compact manifold with a regular contact structure, and showed that it is a principal fiber bundle over a symplectic manifold with a structural group $T^{1}$ (=1-dimensional torus group). Especially it has been shown that a compact, simply connected homogeneous contact manifold is a principal $T^{1}$-bundle over a homogeneous Kählerian manifold.

If we take a contact manifold $M$, then we can provide it a contact metric structure choosing a suitable metric on $M$. Then for any principal $T^{1}$-bundle $P$ over $M$, we can give an almost complex structure using the contact metric structure on $M$ and an infinitesimal connection on $P$. The integrability condition of this almost complex structure is a question. In this note, we shall study the case when $M$ is moreover compact, simply connected and homogeneous contact manifold, and we shall show that any principal $T^{1}$ bundle $P$ over $M$ has a homogeneous complex structure using the above fibering of Boothby-Wang. Since a compact, simply connected homogeneous contact manifold $M$ has a normal contact metric structure [8], if $P$ is a trivial principal bundle, then the proposition is easily shown taking a flat connection. Therefore it is essential when $P$ is not trivial.

I should like to express my sincere gratitude to Professors S. Sasaki and S. Murakami for their kind guidance and many valuable criticism.

1. Preliminaries. Let $M$ be a compact, simply connected homogeneous manifold. It is known that the Lie group $G$ which acts transitively on $M$ has a compact semi-simple subgroup which acts transitively on $M$. Therefore we can take the first $G$ as a compact semi-simple Lie group, and $M$ is a coset space of $G$ by a closed subgroup $L$. Moreover, we can suppose without loss of generality that the compact, semi-simple Lie group $G$ is simply connected. In fact it is sufficient to take a universal covering group of $G$. Therefore we assume that in the following $G$ is compact and simply connected. In this situation, $L$ is also compact, and $G / L$ is a reductive homogeneous space in the sense of K. Nomizu [1]. If we represent the Lie algebra of the Lie group $G$ and $L$ by $\widehat{G}$ and $\widehat{L}$, then there exists a subspace $\widehat{M}$ of $\widehat{G}$ such that 


$$
\widehat{G}=\widehat{L}+\widehat{M}, \quad \widehat{L} \cap \widehat{M}=(0), \quad[\widehat{L}, \widehat{M}] \subset \widehat{M}
$$

We consider a natural principal fiber bundle $G(G / L, \pi, L)$ with a canonical projection $\pi: G \rightarrow G / L$. Then we have a natural $G$-invariant connection $\omega$ on $G$ in accordance with the above decomposition. We consider in the following that the Lie algebra $\widehat{G}$ of $G$ (composed of all left invariant vector fields on $G$ ) is identified with the tangent space of $G$ at the unit element $e$. In this identification, the differential of the right translation of $G$ by the element $g$ of $G$ can be considered as the adjoint operation of $T_{e}(G)$ by $g$. We denote it by $A_{g}$. The differentiable connection $\omega$ is defined for any element $X \in \widehat{G}$, and $g \in G$,

$$
\omega(X)=X_{\hat{L}}, \quad L_{\vartheta}^{*} \omega=\omega
$$

where $X_{\hat{L}}$ means the $\widehat{L}$-component of $X$. It can be shown that $\omega$ satisfies the two conditions of connection on $G$ :

$$
\begin{array}{lll}
\omega(\bar{g} l)=A_{l}^{-1} \omega(\bar{g}), & \bar{g} \in T_{g}(G), l \in L, \\
\omega(g \bar{l})=l^{-1} \bar{l}, & g \in G, \bar{l} \in T_{l}(L) .
\end{array}
$$
$T^{1}$ by

Next we define a 1 -form $\Psi$ on $G$ using a homomorphism $\mu$ of $L$ onto

$$
\Psi=\mu \cdot \omega
$$

Then $\Psi$ is $G$-invariant and takes value in $\widehat{T}^{1}$. If we restrict $\Psi$ on $G$, then it satisfies the following equations:

$$
\begin{array}{ll}
\Psi A_{l}=\Psi, & l \in L, \\
\Psi(\bar{l})=\mu(\bar{l}), & \bar{l} \in \widehat{L} .
\end{array}
$$

We take a differentiable $T^{1}$-bundle $P$ over $M$. S. Murakami [2] showed that if the Lie group $G$ is compact and simply connected, then any differentiable principal bundle $P$ over a homogeneous space $G / L$ with an abelian structural group $T^{1}$ corresponds to a differentiable homomorphism $\mu: L \rightarrow T^{1}$. $P$ is obtained from $\mu$ and the principal fiber bundle $G(G / L, \pi, L)$ as follows: Let $G \times T^{1}$ be the product space of $G$ and $T^{1}$, and we introduce an equivalence relation $(\sim)$ in $G \times T^{1}$ by the rule

$$
(g, a) \sim\left(g l, \mu(l)^{-1} a\right), \quad l \in L .
$$

Then the manifold $P=G \times T^{1} /(\sim)$ is a differentiable principal bundle space 
over $G / L$ with the structural group $T^{1}$. We take a 1 -form $\Psi$ for this homomorphism $\mu$. If we write the kernel of $\mu$ by $K$, then $K$ is clearly a closed subgroup of $L$ and of course that of $G$. We suppose that the homogeneous space $G / L$ admits a non-trivial principal $T^{1}$-bundle $P$ over it. Then by virtue of the theorem of Murakami cited above, there exists a non-trivial homomorphism $\mu$ of $L$ onto $T^{1}$. This fact is also equivalent to the condition that the compact Lie group $L$ is not semi-simple.

LEMMA 1. The principal fiber bundle $P\left(G / L, p, T^{1}\right)$ is equivalent to the principal fiber bundle $G / K(G / L, p, L / K)$ in a natural fashion.

ProOf. Let $\lambda: G \rightarrow G / K$ be a canonical map which coincides with $\mu$ on $L$. We correspond any element $\lambda(g)$ of $G / K$ to the class $[(g, e)]$ of $P$. This correspondence is well defined, and is clearly onto, one-to-one. We take an element $\lambda(g)$ of $G / K$ and an element $\mu(l)$ of $L / K=T^{1}$. Since $K$ is an invariant subgroup of $L$, we have

$$
R_{\mu(l)} \lambda(g)=\lambda(g l)
$$

where $R_{\mu(l)}$ denotes the right translation on $G / K$ by the structural group $L / K$. Therefore the above correspondence between $G / K$ and $P$ commutes with the right translations of the structural groups, that is,

$$
R_{\mu(l)}[(g, e)]=[(g l, e)] .
$$

This shows that the correspondence $\lambda(g) \rightarrow[(g, e)]$ is a bundle map. Therefore $P$ and $G / K$ have equivalent bundle structures.

Q. E. D.

By virtue of this lemma, we can identify the principal fiber bundle $P\left(G / L, p, T^{1}\right)$ with $G / K(G / L, p, L / K)$. In the following we always take this identification. Since the Lie algebra $\widehat{T}^{1}$ of the 1-dimensional Lie group $T^{1}$ $=L / K$ is the 1 -dimensional real line, we can consider every $\widehat{T}^{1}$-valued mapping as a real valued mapping. Therefore $\Psi$ is a real valued left invariant 1 -form on $G$. The center of $\widehat{L}$ is mapped onto $\widehat{T}^{1}$ by $\mu: \widehat{L} \rightarrow \widehat{T}^{1}$ induced from the homomorphism $L \rightarrow T^{1}$. Therefore we can choose an element $\alpha$ of the center of $\hat{L}$ such as $\mu(\alpha)=1$. Then it holds

$$
\Psi(\alpha)=\mu \cdot \omega(\alpha)=\mu(\alpha)=1
$$

$\widehat{L}$ can be decomposed to the direct sum of the Lie algebra $\widehat{K}$ of $K$ and the space $\{\alpha\}$ generated by $\alpha$. 
2. Homogeneous almost contact manifold. Suppose $M$ be a compact, simply connected manifold on which a compact simply connected Lie group $G$ acts transitively. Then there exists an isotropic subgroup $L$ of $G$ and $M$ is diffeomorphic with $G / L$. As in the previous section, we have a 1 -form $\Psi$. Furthermore we assume that $M$ admits a $G$-invariant almost contact structure $(\phi, \xi, \eta)$. These tensors are of types $(1,1),(1,0)$ and $(0,1)$ and satisfy the following relations :

$$
\begin{aligned}
& \eta(\xi)=1, \\
& \phi(\xi)=0, \\
& \phi \phi=-\delta+\eta \otimes \xi .
\end{aligned}
$$

From the projection $\pi: G \rightarrow G / L$ we have the inverse images $\phi^{*}, \eta^{*}$ on $G$

$$
\phi^{*}=\pi^{*} \phi, \quad \eta^{*}=\pi^{*} \eta
$$

$\phi^{*}$ is a $T(M)$-valued $G$-invariant mapping on $T(G)$ and $\eta^{*}$ is a $G$-invariant tensor fields of type $(0,1)$ on $G$. The restrictions of $\phi^{*}$ and $\eta^{*}$ on $\widehat{G}$ satisfy the following relations;

$$
\begin{aligned}
& \phi^{*} A_{l}=\phi^{*}, \quad \eta^{*} A_{l}=\eta^{*}, \quad l \in L, \\
& \phi^{*}(\widehat{L})=\eta^{*}(\widehat{L})=0 .
\end{aligned}
$$

We define an endomorphism $I: \widehat{G} \rightarrow \widehat{G} \quad$ by

$$
\begin{aligned}
& \pi(I X)=\phi^{*}(X)+\Psi(X) \xi, \\
& \omega(I X)=-\eta^{*}(X) \alpha .
\end{aligned}
$$

Then we have

$$
\Psi(I X)=-\eta^{*}(X)
$$

THEOREM. 1. The endomorphism I satisfies the following properties:

(i) $\quad I X=0$ if and only if $X \in \widehat{K}$,

(ii) $I^{2} X \equiv-X \quad(\bmod \widehat{K}), \quad X \in \widehat{G}$,

(iii) $I A_{k} X \equiv A_{k} I X \quad(\bmod \widehat{K}), \quad k \in K, \quad X \in \widehat{G}$,

(iv) $\quad I \alpha \in \widehat{M}, \quad A_{l} I \alpha=I \alpha, \quad l \in L$. 
Therefore I defines a homogeneous almost complex structure on $G / K$.

Proof. (i). If $X \in \hat{K}$, then $\pi(I X)=0, \omega(I X)=0$. Therefore $I X=0$. Conversely if $I X=0$ for $X \in \widehat{G}$, then we have $\pi X=0$, and so $X \in \widehat{L}$. We denote $X_{\alpha}$ the $\{\alpha\}$-component of $X$, with respect to the decomposition $\widehat{L}=\widehat{K}+\{\alpha\}$. Then $\pi(I X)=\Psi\left(X_{\alpha}\right) \xi=0$, therefore $X_{\alpha}=0$. This shows that $X$ belongs to $\hat{K}$.

(ii). For any $X \in \widehat{G}$, we have

$$
\begin{aligned}
& \pi\left(I^{2} X\right)=\phi(\pi I X)+\Psi(I X) \xi=-\pi(X), \\
& \Psi\left(I^{2} X\right)=-\eta(\pi I X)=-\Psi(X) .
\end{aligned}
$$

On the other hand $X$ satisfies $\pi(X)=\Psi(X)=0$ if and only if $X \in \widehat{K}$. Therefore from the above two equalities, we have

$$
I^{2} X \equiv-X(\bmod \widehat{K}) \text {. }
$$

(iii). Since $\phi, \xi$ and $\eta$ are $G$-invariant, we have for $k \in K, X \in \widehat{G}$,

$$
\begin{aligned}
\pi\left(I A_{k} X\right) & =\phi\left(L_{k}^{-1} \pi X\right)+\Psi(X) L_{k}^{-1} \xi \\
& =L_{k}^{-1}\{\phi(\pi X)+\Psi(X) \xi\} \\
& =L_{k}{ }^{-1} \pi(I X)=\pi\left(A_{k} I X\right), \\
\Psi\left(I A_{k} X\right) & =-\eta\left(\pi A_{k} X\right)=-\eta(\pi X), \\
\Psi\left(A_{k} I X\right) & =-\eta(\pi X) .
\end{aligned}
$$

Therefore it holds $I A_{k} X \equiv A_{k} I X(\bmod \widehat{K})$ for all $X \in \widehat{G}$. We see more precisely that in this equation the element of $K$ can be replaced with the element of $L$, since the above three equations hold when we take $l \in L$ in place of $k \in K$.

(iv). If we put $\beta=I \alpha$, then

$$
\begin{aligned}
& \pi(\beta)=\phi(\pi \alpha)+\Psi(\alpha) \xi=\xi \neq 0, \\
& \omega(\beta)=-\eta(\pi \alpha)=0 .
\end{aligned}
$$

Therefore $\beta$ is non-zero and belongs to $\widehat{M}$. As the Lie algebra $\widehat{G}$ is reductive we have $A_{l} \beta \in \widehat{M}$ for $l \in L$. On the other hand, we have seen that $I A_{l} \alpha \equiv A_{l} I \alpha(\bmod \hat{K})$ for $l \in L$. As $\alpha$ is an element of the center of $\hat{L}$, it 
holds that $A_{l} \alpha=\alpha$ for $l \in L$. Therefore we have $A_{l} \beta \equiv \beta(\bmod \widehat{K})$. Therefore $A_{l} \beta-\beta$ belongs to both $\widehat{M}$ and $\widehat{K}$, which means

$$
A_{l} \beta=\beta, \quad l \in L .
$$

The conditions (i) (iii) of this theorem show that any $T^{1}$-bundle $P$ over $M=G / L$ has a homogeneous almost complex structure.

Conversely, we take a $T^{1}$-bundle $P$ over a compact simply connected homogeneous manifold $M=G / L$ and suppose that $P=G / K$ admits a homogeneous almost complex structure. Then there exists an endomorphism $I: \widehat{G} \rightarrow \widehat{G}$ which satisfies the conditions (i) (iii) of Theorem 1. If there exists an element $\alpha \in \widehat{L}$ such that

$$
\beta=I \alpha \in \widehat{M}, \quad A_{l} \beta=\beta \quad \text { for } \quad l \in L,
$$

then the base manifold $G / L$ admits a homogeneous almost contact structure. In fact, by virtue of (iv), the non-zero vector $\pi \beta \in T_{x_{0}}(G / L)$ can be extended to a $G$-invariant vector field $\xi$ over $G / L$ which takes value $\pi \beta$ at $x_{0}=\pi(e)$ $\epsilon G / L$. For this purpose it is sufficient to define $\xi_{x}=L_{g} \xi_{x_{0}}$ for $x=L_{\theta} x_{0}$. Other tensors $\phi, \eta$ over $G / L$ can be defined as follows: for any vector $\bar{x} \in T_{x_{0}}(G / L)$, taking a lift $X$ of $\bar{x}$ with respect to the connection $\omega$ on $G(G / L, \pi, L)$ (see $\S 1$ ), we define at $x_{0}$

$$
\begin{aligned}
& \phi(\bar{x})=\pi(I X), \\
& \eta(\bar{x})=-\Psi(I X) .
\end{aligned}
$$

For any $x=L_{g} x_{0} \in G / L$, we define $\phi_{x}=L_{g} \phi_{x_{0}} L_{g}^{-1}, \eta_{x}=\eta_{x_{0}} \cdot L_{g}^{-1}$. Then for a lift $X \in \widehat{G}$ of a vector $\bar{x} \in T_{x_{0}}(G / L), X$ belongs to $\widehat{M}$ and so the lift of $L_{l} \bar{x} \in T_{x_{0}}(G / L)$ is $A_{l} X \in \widehat{M}$ for $l \in L$. Therefore we have by virtue of (i) and (iii)

$$
\begin{gathered}
L_{l}^{-1} \phi\left(L_{l} \bar{x}\right)=L_{l}^{-1} \pi\left(I A_{l} X\right)=L_{l}^{-1} \pi\left(A_{l} I X\right)=\pi(I X)=\phi(\bar{x}), \\
\eta\left(L_{l} \bar{x}\right)=-\Psi\left(I A_{l} X\right)=-\Psi\left(A_{l} I X\right)=\eta(\bar{x}) .
\end{gathered}
$$

The tensors $\phi, \eta$ are well-defined, and clearly are $G$-invariant. Next for any element $X$ of $\hat{G}$, we have $\pi\left(I X_{\hat{L}}\right)=\Psi(X) \xi$. Since the element $X_{\hat{\mu}}$ is a lift of a vector in $T_{x_{0}}(G / L)$, we see that

$$
\phi\left(\pi X_{\hat{\mu}}\right)=\pi I\left(X-X_{\hat{L}}\right)=\pi(I X)-\Psi(X) \xi .
$$


Therefore

$$
\pi(I X)=\phi(\pi X)+\Psi(X) \xi
$$

In a similar way

$$
\Psi(I X)=-\eta(\pi X)
$$

for any $X \in \widehat{G}$. Since the lift of a vector $\phi \bar{x}$ is $I X-\Psi(X) \beta$ when $X$ is a lift of $\bar{x} \in T_{x_{0}}(G / L)$, using these equations and the condition (ii), we see

$$
\begin{gathered}
\eta(\xi)=1, \quad \eta(\phi \bar{x})=0, \quad \phi(\xi)=0, \\
\phi^{2}(\bar{x})=-\bar{x}+\eta(\bar{x}) \xi .
\end{gathered}
$$

Therefore $(\phi, \xi, \eta)$ admits a homogeneous almost contact structure on $M$. This proves the following theorem.

THEOREM 2. Let $M$ be a compact simply connected homogeneous manifold. If a principal $T^{1}$-bundle $P$ over $M$ admits a homogeneous almost complex structure which can be defined by an endomorphism $I: \widehat{G} \rightarrow \widehat{G}$ satisfying the condition (iv), then $M$ admits a homogeneous almost contact structure.

We can decompose $\widehat{G}=\widehat{L}+\widehat{M}$ as $\widehat{L}=\widehat{K}+\{\alpha\}, \widehat{M}=\{\beta\}+\dot{\widehat{M}}^{\prime}$,

$$
\widehat{G}=\widehat{K}+\{\alpha\}+\{\beta\}+\widehat{M}^{\prime}, \quad \text { (direct sum) }
$$

where $\{\boldsymbol{\beta}\}$ denotes the 1-dimensional subspace generated by $\beta$. By virtue of Theorems 1 and 2, a compact simply connected homogeneous space $G / L$ admits a homogeneous almost contact structure when and only when there exists an endomorphism of $\widehat{G}$ whose component is

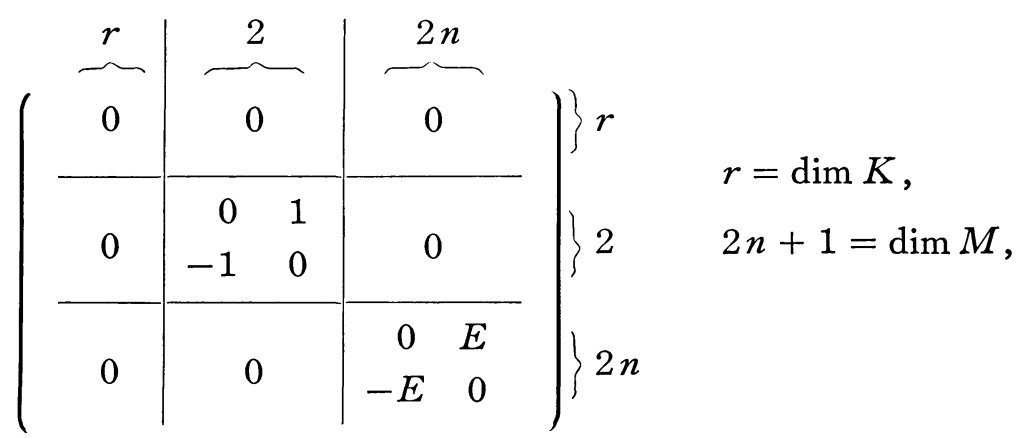

for an adequate basis of $\widehat{G}$. 
3. Homogeneous contact manifold. Let $M$ be a compact, simply connected homogeneous contact manifold. Then $M$ is a coset space of a compact and simply connected Lie group $G$ by an isotropy subgroup $L$. Let $\eta$ be the contact form on $M$ such that $\eta \wedge(d \eta)^{n} \neq 0$ on $M$. Then we shall prove the following

THEOREM 3. There exists on $M$ a homogeneous almost contact metric structure which associates to the homogeneous contact form $\eta$.

Proof. As $M$ is a compact homogeneous manifold $G / L, M$ admits a Riemannian metric which is invariant by the left operation of $G$. We take such a $G$-invariant metric $h$. Since the 2 -form $d \eta$ is skew-symmetric and has rank $2 n$, we can adopt the method of Y. Hatakeyama [7]. Consider two $G$-invariant distributions $D_{1}, D_{2}$ at each point of $M$ :

$$
\begin{aligned}
& D_{1}(x)=\left\{\bar{x} \in T_{x}(M) ; \eta(\bar{x})=0\right\}, \\
& D_{2}(x)=\left\{\bar{x} \in T_{x}(M) ; d \eta\left(\bar{x}, T_{x}(M)\right)=0\right\} .
\end{aligned}
$$

Then $D_{1}$ and $D_{2}$ span the whole tangent space $T_{x}(M)$ and are complement to each other. Therefore we can take at each point $x$ of $M$ a coordinate neighbourhood $U$ and orthonormal frames $e_{1}, e_{2}, \cdots, e_{2 n}, e_{\Delta}$ with respect to the metric $h$, such that $e_{1}, \cdots, e_{2 n} \in D_{1}$ and $e_{\Delta} \in D_{2}$. Now for any $x \in M$, $g \in G$, we put $y=L_{g} x \in M$. Then we can take open neighbourhoods $U$ of $x$ and $V$ of $y$ such that $L_{g} U \subset V$ and they have the above adapted frames. Since for the element $g$ of $G, L_{g}$ is an isometric transformation, it has with respect to the adapted frame the component

$$
L_{o}=\left(\begin{array}{cc}
A_{V}^{T} & 0 \\
0 & 1
\end{array}\right) \text {, }
$$

where $A_{V}^{U}$ is an orthogonal matrix of degree $2 n$. On the other hand, the components of 2 -form $d \eta$ on $U$ are

$$
\psi_{U}=\left(\begin{array}{cc}
\psi_{U}^{\prime} & 0 \\
0 & 0
\end{array}\right)
$$

where $\psi_{U}^{\prime} \in G L(2 n)$. We can decompose the regular matrix $\psi_{U}^{\prime}$ as the product of $\phi_{U}^{\prime} \in O(2 n)$ and $g_{U}^{\prime} \in H(2 n)$ where $O(2 n)$ and $H(2 n)$ denote the orthogonal group of degree $2 n$ and the set of all positive definite symmetric matrices of degree $2 n$. Since $d \eta$ is $G$-invariant, we have $L_{g} \psi_{U}=\psi_{V} L_{g}$, therefore 


$$
A_{V}^{U} \psi_{U}^{\prime}=\psi_{V}^{\prime} A_{V}^{T}
$$

And so

$$
A_{V}^{U} \phi_{U}^{\prime}{ }^{t} A_{V}^{U} A_{V}^{U} g_{U}^{\prime}{ }^{t} A_{V}^{U}=\phi_{V}^{\prime} g_{V}^{\prime}
$$

From the uniqueness of the decomposition $G L(2 n) \rightarrow O(2 n) \times H(2 n)$ and from the fact that $A_{V}^{U}$ is an orthogonal matrix, we have

$$
\begin{aligned}
& \phi_{V}^{\prime}=A_{V}^{U} \phi_{U}^{\prime}{ }^{t} A_{V}^{U}, \\
& g_{\Gamma^{\prime}}^{\prime}=A_{V}^{U} g_{U}^{\prime}{ }^{t} A_{V}^{U} .
\end{aligned}
$$

Therefore, for the tensors $g$ and $\phi$ whose components are

$$
g_{U}=\left(\begin{array}{cc}
g_{U}^{\prime} & 0 \\
0 & 1
\end{array}\right), \quad \phi_{U}=\left(\begin{array}{cc}
\phi_{U}^{\prime} & 0 \\
0 & 0
\end{array}\right) \text {, }
$$

it holds that $L_{g} g_{U}=g_{V} L_{g}, L_{g} \phi_{U}=\phi_{V} L_{g}$. This shows that the almost contact $\begin{array}{ll}\text { metric structure }(\phi, \xi, \eta, g) \text { is } G \text {-invariant. Q.E.D. } & \text { Q. }\end{array}$

By virtue of this Theorem 3 , we can consider $G / L$ as a homogeoeous almost contact manifold. We take a non-trivial $T^{1}$-bundle over a compact simply connected homogeneous contact manifold $M=G / L$. Then by virtue of $\S 1$, this principal bundle space can be identified with the homogeneous space $G / K$ where $K$ is the kernel of the homomorphism $\mu: L \rightarrow T^{1}$. Let $K_{0}$ be the identity component of $K$. As $G$ is simply connected, $P_{0}=G / K_{0}$ is a universal covering space of $G / K=P$. Moreover we know owing to BoothbyWang [3] that a compact simply connected homogeneous contact manifold $G / L$ is a principal $T^{1}$-bundle over a homogeneous Kähler manifold $G / H$. The fiber $H / L$ is a 1-parameter torus group $T^{1}$ which is generated at each point of $M$ by an associated direction field $\xi$ of the contact form $\eta$.

LEMMA 2. The Lie algebra $\hat{H}$ of the Lie subgroup $H$ is spanned by $\hat{L}$ and $\beta$.

ProOF. Since $\operatorname{dim}(G / H)=2 n$, we have $\operatorname{dim} \hat{H}=\operatorname{dim} \hat{L}+1$. We take an element $\gamma \in \widehat{G}$ which spans $\hat{H}$ in company with $\hat{L}$. The Lie algebra of $T^{1}=H / L$ which can be considered as the image of $\hat{H}$ by the natural projection $\pi: G \rightarrow G / L$ is isomorphic with the vertical subspace of a tangent space to $G / L$ at any point of $G / L$ (with respect to the connection $\eta$ ). The latter 
is spanned by $\xi$. Therefore we have $\{\pi \gamma\}=\{\pi \beta\}$, and $\gamma$ is equal to $\beta$ modulo $\widehat{L}$. This proves our Lemma.

LEMMA 3. The homogeneous space $H / K_{0}$ is a complex 1-dimensional torus.

PROOF. By virtue of the condition (iv) of Theorem 1 , we know $[\hat{L}, \beta]$ $=0$. Therefore $\widehat{H}=\widehat{L}+\{\beta\}$ is contained in the centralizer of $\beta$ in $\widehat{G}$. Since $[\widehat{H}, \hat{H}]=[\widehat{L}, \widehat{L}] \subset \widehat{K}$, the Lie algebra $\widehat{K}$ is an ideal of $\hat{H}$, and the quotient subalgebra $\hat{H} / \widehat{K}$ is abelian. Consequently the compact connected abelian Lie group $H / K_{0}$ associating to the Lie algebra $\hat{H} / \hat{K}$ is isomorphic with the 2dimensional torus $T^{2}$. However we can verify easily that the endomorphism $I: \widehat{G} \rightarrow \widehat{G}$ gives a homogeneous almost complex structure on $H / K_{0}$. Since the generators $\alpha, \beta$ of the Lie algebra $\widehat{H} / \widehat{K}$ satisfy $I \alpha=\beta, I \beta=-\alpha$, we have

$$
N(\alpha, \beta)=[\alpha, \beta]+I[I \alpha, \beta]+I[\alpha, I \beta]-[I \alpha, I \beta]=0
$$

Therefore this almost complex structure on $H / K_{0}$ is integrable, and so $H / K_{0}$ is a complex 1-dimensional torus.

From this Lemma, we can give the structure of a complex Lie algebra of $H / K_{0}$ by the relation

$$
\beta=\sqrt{-1} \alpha
$$

Next we consider the integrability condition of the almost complex structure defined in Theorem 1 . As the base space $G / H$ of the fibering of Boothby-Wang is a homogeneous Hodge manifold, we can take a normal contact metric structure $(\phi, \xi, \eta, g)$ on $G / L$ associated to the contact form $\eta$ [8]. In this case, for the $G$-invariant complex structure $J_{0}$ of $G / H$, it holds

$$
q \phi=J_{0} q
$$

where $q$ denotes the natural projection $q: G / L \rightarrow G / H$. As $\eta$ and the metric $g$ is $G$-invariant, $\phi$ is also $G$-invariant. We can give to a principal bundle $P_{0}=G / K_{0}\left(G / L, p, L / K_{0}\right)$ an endomorphism $I$ defined as in Theorem 1 . A $G$-invariant almost complex structure $I_{0}$ of $G / K_{0}$ can be defined by

$$
\lambda I=I_{0} \lambda,
$$

where $\lambda$ denotes the natural projection $G \rightarrow G / K_{0}$. 
Now we consider the principal bundle $G / K_{0}\left(G / H, r, H / K_{0}\right)$. The projection $r$ is the product of the projections $p$ and $q$. Then we have

$$
r I_{0}=J_{0} r
$$

As $G / H$ is a reductive homogeneous space, we can take a natural $G$-invariant connection $\widetilde{\omega}_{0}$ on $G(G / H, H)$ with some reduction of $G / H$. Then on a principal bundle $G / K_{0}\left(G / H, r, H / K_{0}\right)$ there exists a connection $\widetilde{\omega}$ associated to $\widetilde{\omega}_{0}$. Then we have

LEMMA 4. $\widetilde{\omega}$ is of type $(1,0)$ for the almost complex structure $I_{0}$ on $G / K_{0}$, that is, it holds

$$
\widetilde{\omega}\left(I_{0} \bar{u}\right)=\sqrt{-1} \widetilde{\omega}(\bar{u}), \quad \bar{u} \in T\left(G / K_{0}\right) .
$$

PROOF. It is sufficient to prove for the tangent vector at $\lambda(e) \in G / K_{0}$. We take an element $\lambda X$ of $T_{\lambda(e)}\left(G / K_{0}\right)$, where $X$ belongs to $\widehat{G}=T_{e}(G)$. Then

$$
\widetilde{\omega}\left(I_{0} \lambda X\right)=\widetilde{\omega}_{0}(I X)=(I X)_{\hat{H}},
$$

$X_{\hat{H}}$ denotes the $\hat{H}$-component of $X$. As $I \hat{H} \subset \widehat{H}, I \widehat{M}^{\prime} \subset \widehat{M^{\prime}}$, we have

$$
(I X)_{\hat{H}}=I X_{\hat{H}}=\sqrt{-1} \widetilde{\omega}(\lambda X) .
$$

The principal bundle $G / K_{0}\left(G / H, r, H / K_{0}\right)$ is therefore almost complex principal bundle. Since the curvature form of the connection $\widetilde{\omega}$ is $d \widetilde{\omega}$, we have the following proposition by virtue of Theorem 2 of [5].

Proposition. The almost complex structure $I_{0}$ is integrable if and only if the $(0,2)$-component of $d \widetilde{\omega}$ vanishes.

However, with respect to the homogeneous complex structure of a principal bundle space $P$ over $M$, we see that $P$ is covered universally by a $C$ manifold $P_{0}=G / K_{0}$. In fact, since $H / K_{0}$ is an abelian group, the commutator subgroup of $H$ is contained in $K_{0}$. If we denote the semi-simple parts of groups $H$ and $K_{0}$ by $H^{\prime}$ and $K_{0}^{\prime}$, then $K_{0}^{\prime}$ coincides with $H^{\prime}$. For the homogeneous Kählerian $C$-manifold $G / H$, the isotropy subgroup $H$ is a $C$ subgroup of $G$. Therefore $H^{\prime}$ is the semi-simple part of a centralizer of a torus in $G$, and $K_{0}^{\prime}$ is also. This shows that $G / K_{0}$ is a $C$-manifold and $G / K_{0}\left(G / H, H / K_{0}\right)$ is a complex torus bundle [4]. This proves the following 
THEOREM 4. Let $M$ be a compact, simply connected homogeneous contact manifold. Then any principal $T^{1}$-bundle space $P$ over $M$ has a homogeneous complex structure and it is a non-Kählerian complex analytic principal $T^{1}(C)$-bundle over a Kählerian $C$-manifold.

\section{BIBLIOGRAPHY}

[1] K. NomIZU, Invariant affine connections on homogeneous spaces, Amer. Journ. Math., 76(1954), 33-65.

[2] S. MURAKAmI, Sur certains espaces fibrés principaux différentiables et holomorphes, Nagoya Math. Journ., 15(1959), 171-199.

[3] W. M. BoothBy AND H. C. WANG, On contact manifolds, Ann. of Math., 68(1958), 721-734.

[4] J. HANO AND S. KOBAYASHI, A fibering of a class of homogeneous complex manifolds, Trans. Amer. Math. Soc., 94(1960), 233-243.

[5] J. L. Koszul AND B. MALGRANGe, Sur certaines structures fibrées complexes, Arkiv. der Math., 9(1958), 102-109.

[6] S. SASAKI AND Y. HATAKEYAMA, On differentiable manifolds with certain structures which are closely related to almost contact structures II, Tôhoku Math. Journ., 13 (1961), 281-294.

[7] Y. HATAKEYAMA, On the existance of Riemann metrics associated with a 2-form of rank 2 r, Tôhoku Math. Journ., 14(1962), 161-166.

[8] Some notes on differentiable manifolds with almost contact structures, Tôhoku Math. Journ., 15(1963), 176-181.

[9] Y. OGAWA, Some properties on manifolds with almost contact structures, Tôhoku Math. Journ., 15(1963), 148-161.

TôHOKU UNIVERSITY. 